\title{
Holographic Chaplygin DGP cosmologies
}

\author{
M.R. Setare * \\ Department of Science, Payame Noor University. Bijar, Iran
}

\begin{abstract}
In the present paper, we present an extra dimensions inspired model that is built on the DGP brane-world scenario, then we take the dark energy component on the brane to be a Chaplygin gas. After that we consider a holographic model of Chaplygin gas in the framework of DGP cosmology. We show that the holographic Chaplygin gas can mimic a phantom fluid and cross the phantom divide in a DGP brane-world setup.
\end{abstract}

*E-mail: rezakord@ipm.ir 


\section{Introduction}

The type Ia supernova observations suggests that the universe is dominated by dark energy (DE) with negative pressure which provides the dynamical mechanism of the accelerating expansion of the universe $[1,2,3]$. The strength of this acceleration is presently matter of debate, mainly because it depends on the theoretical model implied when interpreting the data.

An approach to the problem of DE arises from the holographic principle that states that the number of degrees of freedom related directly to entropy scales with the enclosing area of the system. It was shown by 'tHooft and Susskind [4] that effective local quantum field theories greatly overcount degrees of freedom because the entropy scales extensively for an effective quantum field theory in a box of size $L$ with UV cut-off $\Lambda$. As pointed out by [5], attempting to solve this problem, Cohen et al showed [6] that in quantum field theory, short distance cut-off $\Lambda$ is related to long distance cut-off $L$ due to the limit set by forming a black hole. In other words the total energy of the system with size $L$ should not exceed the mass of the same size black hole, i.e. $L^{3} \rho_{\Lambda} \leq L M_{p}^{2}$ where $\rho_{\Lambda}$ is the quantum zero-point energy density caused by UV cut-off $\Lambda$ and $M_{P}$ denotes the Planck mass $\left(M_{p}^{2}=1 / 8 \pi G\right)$. The largest $L$ is required to saturate this inequality. Then its holographic energy density is given by $\rho_{\Lambda}=3 c^{2} M_{p}^{2} / L^{2}$ in which $c$ is a free dimensionless parameter and coefficient 3 is for convenience. As an application of the holographic principle in cosmology, it was studied by [7] that the consequence of excluding those degrees of freedom of the system which will never be observed by the effective field theory gives rise to IR cut-off $L$ at the future event horizon. Thus in a universe dominated by DE, the future event horizon will tend to a constant of the order $H_{0}^{-1}$, i.e. the present Hubble radius. On the basis of the cosmological state of the holographic principle, proposed by Fischler and Susskind [8], a holographic model of dark Energy (HDE) has been proposed and studied widely in the literature $[9,10]^{1}$ In HDE, in order to determine the proper and well-behaved system's IR cut-off, there are some difficulties that must be studied carefully to get results adapted with experiments that claim our universe has accelerated expansion. For instance, in the model proposed by [9], it is discussed that considering the particle horizon, as the IR cut-off, the HDE density reads

$$
\rho_{\Lambda} \propto a^{-2\left(1+\frac{1}{c}\right)}
$$

that implies $w>-1 / 3$ which does not lead to an accelerated universe. Also it is shown in [12] that for the case of closed universe, it violates the holographic bound.

The problem of taking apparent horizon (Hubble horizon) - the outermost surface defined by the null rays which instantaneously are not expanding, $R_{A}=1 / H$ - as the IR cut-off in the flat universe was discussed by Hsu [13]. According to Hsu's argument, employing the Friedmann equation $\rho=3 M_{P}^{2} H^{2}$ where $\rho$ is the total energy density and taking $L=H^{-1}$ we will find $\rho_{m}=3\left(1-c^{2}\right) M_{P}^{2} H^{2}$. Thus either $\rho_{m}$ or $\rho_{\Lambda}$ behave as $H^{2}$. So the DE results as pressureless, since $\rho_{\Lambda}$ scales like matter energy density $\rho_{m}$ with the scale factor $a$ as $a^{-3}$. Also, taking the apparent horizon as the IR cut-off may result in a constant parameter of state $w$, which is in contradiction with recent observations implying variable $w$ [14]. On the other hand taking the event horizon, as the IR cut-off, gives results compatible with observations for a flat universe.

\footnotetext{
${ }^{1} \mathrm{~A}$ very recent development is the idea of bulk holographic dark energy. In this proposal, holographic dark energy is accommodated in the framework of braneworld cosmology [11].
} 
It is fair to claim that the simplicity and reasonable nature of HDE provide a more reliable framework for investigating the problem of DE compared with other models proposed in the literature[15, 16, 17]. For instance the coincidence or "why now?" problem is easily solved in some models of HDE based on this fundamental assumption that matter and holographic dark energy do not conserve separately, but the matter energy density decays into the holographic energy density [18].

In a very interesting paper Kamenshchik, Moschella, and Pasquier [19] have studied a homogeneous model based on a single fluid obeying the Chaplygin gas equation of state

$$
P=\frac{-A}{\rho}
$$

where $P$ and $\rho$ are respectively pressure and energy density in comoving reference frame, with $\rho>0 ; A$ is a positive constant. This equation of state has raised a certain interest [20] because of its many interesting and, in some sense, intriguingly unique features. Some possible motivations for this model from the field theory points of view are investigated in [21]. The Chaplygin gas emerges as an effective fluid associated with d-branes [22] and can also be obtained from the Born-Infeld action [23].

An alternative way of explaining the observed acceleration of the late universe is to modify gravity at large scales. In the present paper, we present an extra dimensions inspired model that is built on the DGP braneworld scenario [24], in this framework, existence of a higher dimensional embedding space allows for the existence of bulk or brane matter which can certainly influence the cosmological evolution on the brane. Here we take the dark energy component on the brane to be a Chaplygin gas. The DGP model has a large scale/low energy effect of causing the expansion rate of the universe to accelerate. We assume that the relation $\rho_{\Lambda}=3 c^{2} M_{p}^{2} / L^{2}$ still holds in the DGP model, then we suggest a correspondence between the holographic dark energy scenario and the Chaplygin gas dark energy model in the framework of DGP cosmology. According to the result of [25] a Chaplygin gas can mimic a phantom fluid and cross the phantom divide in a DGP braneworld setup. Our calculation show, taking $\Omega_{\Lambda}=0.73$ for the present time, it is possible to have $w_{\Lambda}$ crossing -1 . This implies that one can generate phantom-like equation of state from the holographic dark energy model in flat universe in the DGP cosmology framework. We show this holographic description of the Chaplygin gas dark energy in FRW universe and reconstruct the potential and the dynamics of the scalar field which describe the Chaplygin cosmology. We show in order that the holographic dark energy model, which is inspired by quantum gravity, to be consistent with the Chaplygin gas model of dark energy in the DGP framework, one must be careful to satisfy the corresponding constraints which given by equations. (30), (31), i.e, the scalar field which is the origin of Chaplygin gas has to follow potential (30) and equation (31), in order for the two models to be compatible and thus efficiently unified.

\section{Holographic dark energy and Chaplygin gas in DGP braneworld}

We consider a DGP- braneworld model where the dark energy component on the brane is given by a Chaplygin gas, and with an extra CDM component [25]. In the DGP model, it is supposed that a 3 -dimensional brane is embedded in 5-dimensional spacetime. This 
model predicts that 4-dimensional Einstein gravity is a short-distance phenomenon with deviations showing up at large distances. The DGP model includes a length scale below which the potential has usual Newtonian form and above which the gravity becomes 5dimensional. The cross over scale between the 4-dimensional and 5-dimensional gravity is given by

$$
r_{c}=\frac{M_{P}^{2}}{2 M_{5}^{2}}
$$

where $M_{5}$ is the fundamental scale of gravity in five dimension. For the spatially flat Robertson-Walker universe

$$
d s^{2}=-d t^{2}+a(t)^{2}\left(d r^{2}+r^{2} d \Omega^{2}\right) .
$$

the first Friedmann equation is given by

$$
3 H^{2}=\rho_{m}+\rho_{e f f}
$$

here we take $M_{P}^{2}=1$, and $\rho_{m}$ is the energy density of CDM and effective energy density is given by

$$
\rho_{e f f}=\rho-\frac{3 H}{r_{c}} .
$$

where $\rho$ is the energy density of Chaplygin gas. Inserting the equation of state (2) into the relativistic energy conservation equation, leads to a density evolving as

$$
\rho=\sqrt{A+\frac{B}{a^{6}}}
$$

where $B$ is an integration constant.

Now following [26] we assume that the origin of the dark energy is a scalar field $\phi$, so

$$
\begin{aligned}
& \rho_{\phi}=\frac{1}{2} \dot{\phi}^{2}+V(\phi)=\sqrt{A+\frac{B}{a^{6}}} \\
& P_{\phi}=\frac{1}{2} \dot{\phi}^{2}-V(\phi)=\frac{-A}{\sqrt{A+\frac{B}{a^{6}}}}
\end{aligned}
$$

Then, one can easily derive the scalar potential and kinetic energy term as

$$
\begin{gathered}
V(\phi)=\frac{2 a^{6}\left(A+\frac{B}{a^{6}}\right)-B}{2 a^{6} \sqrt{A+\frac{B}{a^{6}}}} \\
\dot{\phi}^{2}=\frac{B}{a^{6} \sqrt{A+\frac{B}{a^{6}}}}
\end{gathered}
$$

Now we suggest a correspondence between the holographic dark energy scenario and the Chaplygin gas in DGP braneworld. The holographic dark energy scenario reveals the dynamical nature of the vacuum energy. When taking the holographic principle into account, the vacuum energy density will evolve dynamically. On the other hand, the scalar field dark energy models are often viewed as effective description of the underlying theory of dark energy. We are now interested in that if we assume the holographic vacuum 
energy scenario as the underlying theory of dark energy, how the scalar field model can be used to effectively describe it.

Our choice for holographic dark energy density is

$$
\rho_{\Lambda}=\frac{3 c^{2}}{R_{h}^{2}}
$$

where $c$ is a constant, and $R_{h}$ is the future event horizon given by

$$
R_{h}=a \int_{t}^{\infty} \frac{d t}{a}=a \int_{a}^{\infty} \frac{d a}{H a^{2}}
$$

The critical energy density, $\rho_{c r}$, is given by following relation

$$
\rho_{c r}=3 H^{2}
$$

Now we define the dimensionless dark energy as

$$
\Omega_{\Lambda}=\frac{\rho_{\Lambda}}{\rho_{c r}}=\frac{c^{2}}{R_{h}^{2} H^{2}}
$$

Using definition $\Omega_{\Lambda}$ and relation $(14), \dot{R}_{h}$ gets:

$$
\dot{R}_{h}=R_{h} H-1=\frac{c}{\sqrt{\Omega_{\Lambda}}}-1
$$

By considering the definition of holographic energy density $\rho_{\Lambda}$, and using Eqs.( 15), (16) one can find:

$$
\dot{\rho_{\Lambda}}=-2 H\left(1-\frac{\sqrt{\Omega_{\Lambda}}}{c}\right) \rho_{\Lambda}
$$

Substitute this relation into following equation

$$
\dot{\rho}_{\Lambda}+3 H\left(1+w_{\Lambda}\right) \rho_{\Lambda}=0
$$

we obtain

$$
w_{\Lambda}=-\left(\frac{1}{3}+\frac{2 \sqrt{\Omega_{\Lambda}}}{3 c}\right)
$$

A direct fit of the present available SNe Ia data with this holographic model indicates that the best fit result is $c=0.21$ [27]. Recently, by calculating the average equation of state of the dark energy and the angular scale of the acoustic oscillation from the BOOMERANG and WMAP data on the CMB to constrain the holographic dark energy model, the authors show that the reasonable result is $c \sim 0.7$ [28]. In the other hand, in the study of the constraints on the dark energy from the holographic connection to the small $l$ CMB suppression, an opposite result is derived, i.e. it implies the best fit result is $c=2.1$ [29]. Thus according to these studies $0.21 \leq c \leq 2.1$. Taking $\Omega_{\Lambda}=0.73$ for the present time, in the case of $c=0.21$, we obtain $w_{\Lambda}=-3.04$, in the other hand for $c=2.1$, one can obtain, $w_{\Lambda}=-0.6$. Using Eq.(19), one can see that by considering $c \leq \sqrt{\Omega_{\Lambda}}$ we obtain $w_{\Lambda} \leq-1$. Therefore taking $\Omega_{\Lambda}=0.73$ for the present time, it is possible to have $w_{\Lambda}$ crossing -1 . Also the authors of [25] have recently showed that a Chaplygin gas can mimic a phantom fluid and cross the phantom divide in a DGP brane-world setup. 
If we establish the correspondence between the holographic dark energy and effective energy density in DGP braneworld

$$
\frac{3 c^{2}}{R_{h}^{2}}=\sqrt{A+\frac{B}{a^{6}}}-\frac{3 H}{r_{c}} .
$$

then using Eqs.(15),(20) we have

$$
B=a^{6}\left[9\left(\Omega_{\Lambda} H^{2}+\frac{H}{r_{c}}\right)^{2}-A\right]
$$

Now we define the effective equation of state parameter $w_{\text {eff }}$ as [25]

$$
1+w_{e f f}=\frac{-\dot{\rho}_{e f f}}{3 H \rho_{e f f}}
$$

Using Eqs. (2), (6) and following continuity equation for Chaplygin gas

$$
\dot{\rho}+3 H(\rho+P)=0
$$

one can obtain following equation

$$
\dot{\rho}_{e f f}=\frac{9 H H_{0}^{2}(1+Z)^{3}}{H+H_{0} \sqrt{\Omega_{r c}}}\left[\Omega_{m} H_{0} \sqrt{\Omega_{r c}}-H\left(1-\frac{A}{\rho_{0}^{2}}\right) \Omega(1+Z)^{3}\left[\frac{A}{\rho_{0}^{2}}+\left(1-\frac{A}{\rho_{0}^{2}}\right)(1+Z)^{6}\right]^{-1 / 2}\right]
$$

where the subscript 0 corresponds to the current value of a given quantity, $Z=(1 / a)-1$ is the redshift of the universe, and

$$
\Omega_{m}=\frac{\rho_{m 0}}{3 H_{0}^{2}}, \quad \Omega=\frac{\rho_{0}}{3 H_{0}^{2}}, \quad \Omega_{r c}=\frac{1}{4 r_{c}^{2} H_{0}^{2}} .
$$

Using the correspondence between the holographic dark energy and effective energy density in DGP braneworld we claim that $w_{\text {eff }}=w_{\Lambda}$, as we have mentioned above, if $c \leq \sqrt{\Omega_{\Lambda}}$, then $w_{\text {eff }}$ crosses -1 .

Using Eqs.(19), (22), and (24) we can obtain

$$
A=\rho_{0} \frac{\left(d \pm \sqrt{d^{2}+4 e}\right)}{2}
$$

where

$$
d=2+e\left(\frac{1}{(1+Z)^{6}}-1\right)
$$

and

$$
e=\frac{\Omega_{m} H_{0} \sqrt{\Omega_{r c}}}{H \Omega}-\frac{2 \Omega_{\Lambda} \frac{\sqrt{\Omega_{\Lambda}}}{c} H+H_{0} \sqrt{\Omega_{r c}}}{3 \Omega(1+Z)^{3} H_{0}^{2}}
$$

Substituting $A$ into Eq.(21) we obtain following relation for $B$

$$
B=a^{6}\left[9\left(\Omega_{\Lambda} H^{2}+\frac{H}{r_{c}}\right)^{2}-\rho_{0} \frac{\left(d \pm \sqrt{d^{2}+4 e}\right)}{2}\right]
$$


Now we can rewritten the scalar potential and kinetic energy term as following

$$
\begin{gathered}
V(\phi)=3 H^{2} \Omega_{\Lambda}-\frac{9\left(\Omega_{\Lambda} H^{2}+\frac{H}{r_{c}}\right)^{2}-\frac{\rho_{0}\left(d \pm \sqrt{d^{2}+4 e}\right)}{2}}{6 H^{2} \Omega_{\Lambda}} \\
\dot{\phi}=\left[\frac{9\left(\Omega_{\Lambda} H^{2}+\frac{H}{r_{c}}\right)^{2}-\frac{\rho_{0}\left(d \pm \sqrt{d^{2}+4 e}\right)}{2}}{3 H^{2} \Omega_{\Lambda}}\right]^{1 / 2}
\end{gathered}
$$

Considering $x(\equiv \ln a)$, we have

$$
\dot{\phi}=\phi^{\prime} H
$$

Then derivative of scalar field $\phi$ with respect to $x(\equiv \ln a)$ is as

$$
\phi^{\prime}=\left[\frac{9\left(\Omega_{\Lambda} H^{2}+\frac{H}{r_{c}}\right)^{2}-\frac{\rho_{0}\left(d \pm \sqrt{d^{2}+4 e}\right)}{2}}{3 H^{4} \Omega_{\Lambda}}\right]^{1 / 2}
$$

Consequently, we can easily obtain the evolutionary form of the field

$$
\phi(a)-\phi\left(a_{0}\right)=\int_{0}^{\ln a}\left[\frac{9\left(\Omega_{\Lambda} H^{2}+\frac{H}{r_{c}}\right)^{2}-\frac{\rho_{0}\left(d \pm \sqrt{d^{2}+4 e}\right)}{2}}{3 H^{4} \Omega_{\Lambda}}\right]^{1 / 2} d x
$$

where $a_{0}$ is the present time value of the scale factor.

\section{Conclusions}

A well-studied model of modified gravity is the DGP braneworld model [24] in which our 4-dimensional world is a FRW brane embeded in a 5-dimensional Minkowski bulk. In the other hand within the different candidates to play the role of the dark energy, the Chaplygin gas, has emerged as a possible unification of dark matter and dark energy, since its cosmological evolution is similar to an initial dust like matter and a cosmological constant for late times. Inspired by the fact that the Chaplygin gas possesses a negative pressure, people [30] have undertaken the simple task of studying a FRW cosmology of a universe filled with this type of fluid.

In this paper we have considered a holographic model of Chaplygin gas in the framework of DGP cosmology. We have shown that the holographic dark energy can be described by the scalar field in a certain way. Then a correspondence between the holographic dark energy and Chaplygin gas model of dark energy in the framework of DGP cosmology has been established, and the potential of the holographic scalar field and the dynamics of the field have been reconstructed. We have shown if $c \leq \sqrt{\Omega_{\Lambda}}$, the holographic dark energy model also will behave like a phantom model of dark energy the amazing feature of which is that the equation of state of dark energy component $w_{\Lambda}$ crosses -1 . Hence, we see, the determining of the value of $c$ is a key point to the feature of the holographic dark energy and the ultimate fate of the universe as well.

\section{References}

[1] S. Perlmutter et al, Astrophys. J.517, 565, (1999). 
[2] P. M. Garnavich et al, Astrophys. J, 493, L53, (1998).

[3] A. G. Riess et al, Astron. J. 116, 1009, (1998).

[4] G. 't Hooft, gr-qc/9310026 ; L. Susskind, J. Math. Phys, 36, (1995), 6377-6396.

[5] Y. S. Myung, Phys. Lett. B 610, (2005), 18-22.

[6] A. Cohen, D. Kaplan and A. Nelson, Phys. Rev. Lett 82, (1999), 4971.

[7] K. Enqvist, S. Hannestad and M. S. Sloth, JCAP, 0502, (2005) 004.

[8] W. Fischler and L. Susskind, hep-th/9806039.

[9] M. Li, Phy. Lett. B, 603, 1, (2004).

[10] D. N. Vollic, hep-th/0306149; H. Li, Z. K. Guo and Y. Z. Zhang, astro-ph/0602521; J. P. B. Almeida and J. G. Pereira, gr-qc/0602103; D. Pavon and W. Zimdahl, hepth/0511053; Y. Gong, Phys. Rev., D, 70, (2004), 064029; B. Wang, E. Abdalla, R. K. Su, Phys. Lett., B, 611, (2005); M. R. Setare, Phys. Lett. B644, 99, (2007); M. R. Setare, Phys. Lett. B648, 329, (2007); M. R. Setare, Phys. Lett. B653, 116, (2007).

[11] E. N. Saridakis, Phys. Lett. B 660, 138 (2008);

E. N. Saridakis, JCAP 0804, 020 (2008) ;

E. N. Saridakis, Phys. Lett. B 661, 335 (2008).

[12] R. Easther and D. A. Lowe hep-th/9902088.

[13] S. D. H. Hsu, Phys. Lett. B, 594, 13, (2004).

[14] U. Alam, V. Sahni, T. D. Saini, A. A. Starobinsky, Mon. Not. Roy. Astron. Soc., 354, 275 (2004); D. Huterer and A. Cooray, Phys. Rev., D, 71, 023506, (2005), Y. Wang and M. Tegmark, astro-ph/0501351.

[15] For review on cosmological constant problem: V. Sahni, A. A. Starobinsky, Int. J. Mod. Phys. D9, 373, (2000); P. J. E. Peebles, B. Ratra, Rev. Mod. Phys., 75, 559606, (2003); J. Kratochvil, A. Linde, E. V. Linder, M. Shmakova, JCAP, 0407, 001, (2004).

[16] R. R. Caldwell, R. Dave and P. J. Steinhardt, Phys. Rev. Lett., 80, (1998) 1582; I. Zlater, L. Wang and P. J. Steinhardt, Phys. Rev. Lett., 82, (1999), 896; T. Chiba, gr-qc/9903094; M. S. Turner and M. White Phys. Rev. D, 56, (1997), 4439.

[17] R. R. Caldwell, Phys. Lett. B 545, 23, (2002); R. R. Caldwell, M. Kamionkowsky and N. N. Weinberg, Phys, Rev, Lett, 91, 071301, (2003); S. Nojiri and S. D. Odintsov, Phys. Lett., B 562, (2003), 147; S. Nojiri and S. D. Odintsov, Phys. Lett., B 565, (2003), 1; S. Nojiri and S. D. Odintsov, Phys. Rev., D, 72, 023003, (2005); S. Nojiri, S. D. Odintsov, O. G. Gorbunova, J. Phys., A, 39, 6627, (2006); S. Capozziello, S. Nojiri, S. D. Odintsov, Phys. Lett. B 632, 597, (2006); B. Feng, X. L. Wang, X. M. Zhang Phys. Lett. B607, 35 , (2005); E. O. Kahya, V. K. Onemli, gr-qc/0612026; M. R. Setare, Phys. Lett. B641, 130, (2006). 
[18] B. Gumjudpai, T. Naskar, M. Sami and S. Tsujikawa, JCAP 0506, 007, (2005); E. J. Copeland, M. Sami and S. Tsujikawa, hep-th/0603057; E.Elizalde, S. Nojiri, S.D. Odintsov and P. Wang, hep-th 0502082, Phys. Rev. D71, 103504, (2005); H. Kim, H. W. Lee and Y. S. Myung, Phys. Lett. B 632, 605, (2006); B. Hu, Y. Ling, Phys. Rev. D73 (2006) 123510; M. R. Setare, Phys. Lett. B642, 1, (2006); M. R. Setare, Phys. Lett. B642, 421, (2006); M. R. Setare, Eur. Phys. J. C50, 991, (2007); M. R. Setare, JCAP, 0701, 023, (2007);M. R. Setare, Phys. Lett. B654, 1, (2007).

[19] A. Yu. Kamenshchik, U. Moschella, and V. Pasquier, Phys. Lett. B511, 265, (2001).

[20] D. Bazeia, R. Jackiw, Ann. Phys. 270 (1998) 246; D. Bazeia, Phys. Rev. D 59 (1999) 085007; R. Jackiw, A.P. Polychronakos, Commun. Math. Phys. 207 (1999) 107; N. Ogawa, Phys. Rev. D62, 085023, (2000).

[21] N. Bilic, G.B. Tupper and R.D. Viollier, Phys. Lett. B535 (2002) 17; N. Bilic, G.B. Tupper and R.D. Vio- llier, astro-ph/0207423.

[22] M. Bordemann and J. Hoppe, Phys. Lett. B317 (1993) 315; J.C. Fabris, S.V.B. Gonsalves and P.E. de Souza, Gen. Rel. Grav. 34 (2002) 53.

[23] M.C. Bento, O. Bertolami and A.A. Sen, Phys. Lett. B575 (2003) 172.

[24] G. R. Dvali, G. Gabadadze and M. Porrati, Phys. Lett. B 485 (2000) 208

[25] M. Bouhmadi-Lopez, and R. Lazkoz, arXiv:0706.3896 [astro-ph].

[26] J. D. Barrow, Phys. Lett. B235, 40, (1990).

[27] Q. G. Huang, Y. Gong, JCAP, 0408, (2004),006.

[28] H. C. Kao, W. L. Lee and F. L. Lin, astro-ph/0501487.

[29] J. Shen, B. Wang, E. Abdalla and R. K. Su, hep-th/0412227.

[30] V. Gorini, A. Kamenshchik, U. Moschella and V. Pasquier, gr-qc/0403062. 